\title{
Sensitivity and specificity of the circulating cathodic antigen rapid urine test in the diagnosis of Schistosomiasis mansoni infection and evaluation of morbidity in a low- endemic area in Brazil
}

\author{
Fernanda Teixeira Ferreira ${ }^{[1]}$, Thiago André Fidelis ${ }^{[1]}$, Thiago Almeida Pereira ${ }^{[1],[2]}$, \\ Alba Otoni ${ }^{[1],[3]}$, Leonardo Campos Queiroz ${ }^{[1]}$, Frederico Figueiredo Amâncio ${ }^{[1]}$, \\ Carlos Maurício Antunes ${ }^{[4]}$ and José Roberto Lambertucci ${ }^{[1]}$
}

[1]. Serviço de Doenças Infecciosas e Parasitárias, Departamento de Clínica Médica, Faculdade de Medicina, Universidade Federal de Minas Gerais, Belo Horizonte, MG, Brasil. [2]. Immunopathogenesis section, Laboratory of Parasitic Diseases, National Institute of Allergy and Infectious Diseases, National Institutes of Health, Bethesda, Maryland, USA. [3]. Núcleo de Pesquisas em Epidemiologia e Saúde Coletiva, Universidade Federal de São João del-Rei, Divinópolis, MG, Brasil. [4]. Instituto de Ensino e Pesquisa, Santa Casa de Misericórdia de Belo Horizonte, Belo Horizonte, MG, Brasil.

\begin{abstract}
Introduction: The Kato-Katz technique is the standard diagnostic test for Schistosoma mansoni infection in rural areas. However, the utility of this method is severely limited by the day-to-day variability in host egg excretion in the stool. In high-transmission areas, the point-of-care circulating cathodic antigen (POC-CCA) urine assay has proven to be a reliable test. However, investigations of the reliability of the POC-CCA assay in low-transmission regions are under way. This study aimed to evaluate the sensitivity and specificity of the POC-CCA assay and the morbidity of schistosomiasis in a low-endemic area in Brazil. Methods: Pains City is a low-transmission zone for schistosomiasis. A total of 300 subjects aged 7-76 years were randomly selected for the POC-CCA cassette test. For $S$. mansoni diagnosis, three stool samples on six slides were compared with one urine sample for each subject. The sensitivity and specificity in the absence of a gold standard were calculated using latent class analysis. Clinical examinations and abdominal ultrasounds were performed in 181 volunteers to evaluate morbidity associated with schistosomiasis. Results: The sensitivity and specificity of the Kato-Katz technique were $25.6 \%$ and $94.6 \%$, respectively. By contrast, the sensitivity and specificity of the POC-CCA assay were $68.1 \%$ and $72.8 \%$, respectively. Hepatosplenic schistosomiasis was diagnosed in two patients (1.1\%). Conclusions: Overall, the POC-CCA urine assay proved to be a useful test for diagnosing S. mansoni in a lowendemic area in Brazil. Severe clinical forms of schistosomiasis can be present even in such low-endemic areas.
\end{abstract}

Keywords: Schistosomiasis. Diagnosis. Stool examination. Rapid urine test (POC-CCA). Hepatosplenic schistosomiasis.

\section{INTRODUCTION}

Despite mass chemotherapy with praziquantel, schistosomiasis is a major public health problem. It has been estimated that 240 million people worldwide are infected with schistosomes, with an additional 700 million at risk of infection ${ }^{1}$. Currently, in Brazil, 2 to 6 million individuals are believed to be infected in the states of Minas Gerais, Alagoas, Bahia, Espírito Santo, and Pernambuco, with small focal areas of schistosomiasis in other states ${ }^{2}$. Minas Gerais is currently the largest endemic area. Schistosoma mansoni is present in 533 of 853 municipalities (61\%, comprising 589,906 individuals) $)^{3}$.

Corresponding author: Dr. José Roberto Lambertucci.

e-mail: jrlambertu@gmail.com

Received 21 October 2016

Accepted 16 May 2017
Most infected individuals are asymptomatic; however, in 5\%$10 \%$ of infected individuals, liver periportal fibrosis results in severe disease, including portal hypertension, splenomegaly, esophageal varices, and recurrent hematemesis. Pathologically, dense bands of fibrosis around the portal tracts are typical of schistosomiasis ${ }^{4,5}$.

Ultrasound examinations are an efficient means for diagnosing liver fibrosis and for the differential diagnosis among other chronic liver diseases. Schistosomal periportal fibrosis appears as an echogenic band surrounding the portal vessels from the hilum to the periphery of the liver ${ }^{5,6}$.

The frequency of severe schistosomiasis, i.e., the hepatosplenic form, decreases after mass chemotherapy with schistosomicides. A lower prevalence of the disease in endemic areas leads to a greater difficulty of diagnosis of the disease using parasitological stool examinations ${ }^{7,8}$.

The Kato-Katz stool technique has been used for many years as the gold standard for the diagnosis of schistosomiasis 
in endemic areas ${ }^{9}$. Immunological methods of diagnosing schistosomiasis have also been used with acceptable success ${ }^{10}$.

A single application of the Kato-Katz method underestimates the prevalence of schistosomiasis, particularly when patients have a low parasite burden ${ }^{7,11}$. Therefore, a more sensitive tool such as the circulating cathodic antigen (CCA) test (Rapid Diagnostics, Pretoria, South Africa), which is based on the direct detection of the parasite's CCA in the host's urine, is currently under evaluation.

This rapid diagnostic test [point-of-care circulating cathodic antigen (POC-CCA)] for schistosomiasis is a CCA immunechromatographic (ICT) test-based diagnostic method. The sensitivity and specificity vary with the prevalence and intensity of infection. The majority of studies have been conducted in Africa in highly endemic areas with high-intensity infections and in school-age children ${ }^{12,13}$.

In this study, we evaluated the sensitivity and specificity of the POC-CCA assay in 300 individuals, aged 7-76 years, in a low-endemic area in Brazil. The prevalence of hepatosplenic schistosomiasis was also investigated.

\section{METHODS}

\section{Ethical consideration}

This study was approved by the Human Research Ethical Board of the Federal University of Minas Gerais (No. 48094015.8.0000.5149). Written assent and consent were obtained from the study participants and their parents or guardians.

\section{Study area}

Pains City has 8,351 inhabitants and is $217 \mathrm{~km}$ from Belo Horizonte, the capital of Minas Gerais, Brazil. The area is $418 \mathrm{~km}^{2}$ and the population density is 19 people $/ \mathrm{km}^{2}$; the human development index is 0.783 . Surveys on schistosomiasis were administered to 32,806 individuals in this area between 2000 and 2015. The prevalence of $S$. mansoni infection, as assessed using one stool sample with two smears per person, varied from $2 \%$ to $10 \%$ (mean, $4.5 \%$ ). Of the population examined, $68 \%$ had light infections (1-99 eggs per gram of feces). According to a 2009 report by the World Health Organization (WHO), an area with a prevalence below $10 \%$ and an intensity of infection $<100$ eggs per gram should be considered a lowendemic zone ${ }^{14}$.

\section{Sample selection}

In 2015, four localities in Pains were selected: Matinha, Posto Agropecuário, Vila Crispim, and Alvorada. The inhabitants included 1,637 volunteers aged 7-95 years (mean, 35 years; median, 37 years). The sample size was calculated based on the following parameters: a) an estimated S. mansoni positivity of $10 \%$, based on a 2014 survey, and b) an $\alpha$ error of 0.05 and a power of $1-\beta=0.90$. Overall, 300 individuals participated in the study. All households were randomly selected in blocks, and inhabitants of the selected houses were eligible and enrolled in the study.

\section{Stool and urine samples}

Sample collection was performed between September and December 2015 by trained field assistants. Three stool samples obtained on 3 consecutive days and one single midstream urine sample were collected from each person. The investigators visited all households and informed the participants of the details of the research study. Information on age, weight, and height was stored using EpiData Software (EpiData Association, Odense, Denmark).

\section{Stool examination}

Duplicate slides of each stool sample were examined using the Kato-Katz technique. Two trained microscopists read each slide. The analysis consisted of the following procedure: 1 sample/1 slide; 1 sample/2 slides; the first and second samples/4 slides; and the first, second, and third samples/6 slides.

\section{Point-of-care circulating cathodic antigen urine assay}

The urine samples were subjected to a commercially available POC-CCA urine assay kit (batch no. 34159, Rapid Diagnostics, Pretoria, South Africa). The urine samples were stored at $-20^{\circ} \mathrm{C}$ until the assay was performed. The POC-CCA assay was performed as follows: 1 drop of urine was deposited in the testing cassette well. After the urine was fully absorbed, 1 drop of buffer (provided with the POC-CCA test kit) was added, and the test results were read 20 min later. In cases where the control bands did not develop, the test was considered invalid, and the urine sample was retested with a new POC-CCA urine assay kit. Valid tests were scored as negative, trace (weak band), or positive.

\section{Evaluation of morbidity}

In December 2015, our research team revisited Pains to evaluate the morbidity of a sample of volunteers living in the same area in a cross-sectional study. A total of 181 (11\%) participants of 1,637 subjects, aged 7-95 years (mean, 38.9 \pm 21.6 years; $62.1 \%$ male), with a mean weight of $64.6 \pm 17.0 \mathrm{~kg}$ and a mean height of $162.5 \pm 12.5 \mathrm{~cm}$, were subjected to clinical and ultrasound examinations.

\section{Clinical examination}

The 181 participants were subjected to anamnesis and physical examination by one of the authors (Lambertucci JR). Particular attention was given to the abdominal examination; specifically, the right hepatic lobe was examined along the anterior axillary line, and the left hepatic lobe was examined along a line passing through the xiphoid process. The spleen was palpated and measured under the left costal margin during deep inspiration with the patient in the dorsal decubitus position.

\section{Diagnosis of hepatosplenic schistosomiasis}

Hepatosplenic schistosomiasis was diagnosed based on the following criteria: clinical evidence of hepatomegaly and splenomegaly and an ultrasound scan showing characteristic periportal fibrosis of the liver ${ }^{5}$.

\section{Ultrasound examinations}

All 181 volunteers were submitted to an abdominal ultrasound scan using a portable Medison SonoAce 1500 
system with a 3.5-MHz probe (Samsung, Korea) and examined according to the proposed protocol of the WHO for ultrasound assessment of schistosomiasis-related morbidity ${ }^{6,15}$.

\section{Treatment}

All patients with $S$. mansoni infection received a single oral dose of praziquantel given by a physician $(50 \mathrm{mg} / \mathrm{kg}$ of body weight $)^{16}$.

\section{Statistical analysis}

Double-entered data were stored in EpiData 3.1 and analyzed using the Statistical Package for the Social Sciences (SPSS) version 19.0 (IBM, Chicago, IL, USA). Descriptive analyses included the distribution of the frequencies, means, medians, standard deviation, tables, and graphics. The prevalence was determined using the Kato-Katz technique and the POC-CCA assay. The intensity of infection was calculated using the arithmetic mean of eggs in each duplicate according to the Kato-Katz technique. One positive slide was considered a positive test. The positive predictive value (PPV) and negative predictive value (NPV) were also calculated. Likelihood ratios were used to assess the value of performing the diagnostic test. The sensitivity and specificity in the absence of a gold standard were calculated using latent class analysis (LCA). The sensitivity and specificity were estimated for each test using the relationship between the true disease class and the observed test patterns. These analyses were performed using the BayesLatentClassModels (version 1.13 December 2015) ${ }^{17}$. The strength of agreement between the POC-CCA assay and Kato-Katz thick smears was assessed using the kappa statistic $(\kappa)$. McNemar's test was used to compare proportions of paired data and to determine whether discordant pairs were present. Statistical significance was determined at the $5 \%$ level.

\section{RESULTS}

\section{Demographics and study population}

Demographic data from the 300 individuals examined in Pains from August to December 2015 are shown in Table 1. The characteristics of the study population are shown in Figure 1.

\section{Schistosomiasis prevalence}

The prevalence of schistosomiasis using the Kato-Katz technique and the POC-CCA assay is summarized in Table 2.

\section{Infection intensity}

Using the Kato-Katz technique, infection intensity was characterized (in eggs per gram of feces) as light (1-99), moderate (100-399), or heavy ( $\geq 400)$. Results of the POC-CCA urine assay were blindly scored by two investigators as either negative or positive and read as either strong or weak (trace band) responses. Negative results for $S$. mansoni eggs were found in 274 of 300 (91.3\%) stool examinations. In 26 positive stool examinations, the number of eggs per gram of feces using the Kato-Katz method varied from 4 to 1,044 (mean, $75.1 \pm$ 209.7; median, 16.0).

\section{Sensitivity, specificity, likelihood ratio, and predictive value}

We analyzed the sensitivity and specificity using the two methods (Table 3). In the first analysis, we compared the results of the detection of $S$. mansoni eggs in the stool using the KatoKatz method. The sensitivity and specificity of the POC-CCA assay using latent class analysis (LCA) were $68.1 \%$ and $72.8 \%$, respectively, whereas the sensitivity and specificity of the KatoKatz method using LCA were $25.6 \%$ and $94.6 \%$, respectively. The PPV, NPV, and likelihood ratio are summarized in Table 3.

\section{False positives and false negatives}

A total of 67 examinations were positive using the POC-CCA assay and negative using the Kato-Katz technique (22.3\%), whereas 11 examinations were negative using the POC-CCA assay and positive using the Kato-Katz method (3.6\%); these results were significantly different according to McNemar's test $(P$-value $=0.001)$.

\section{Analysis of discordant pairs}

To further analyze the discordant pairs, a new investigation was conducted. One of our investigators (FTF) revisited the area to collect additional stool and urine specimens. The sample size was determined according to the number of discordant

TABLE 1

Demographic data of the 300 individuals selected for the study in Pains, Minas Gerais, Brazil, from August to December 2015.

\begin{tabular}{lcc}
\hline Characteristics & $\begin{array}{c}\text { Study population } \\
\mathbf{n = 3 0 0}\end{array}$ & 土SD \\
\hline Women, n (\%) & $189(63 \%)$ & 15.0 \\
Mean age (years) & 43.9 & 17.2 \\
Mean weight (kg) & 70.5 & 0.1 \\
Mean height (m) & 1.6 & 5.9 \\
Mean BMI & 26.4 & 5.9 \\
\hline
\end{tabular}

SD: standard deviation; BMI: body mass index. 
TABLE 2

Prevalence of schistosomiasis according to the diagnostic method and the number of stool slides examined in Pains, Minas Gerais, Brazil ( $\mathrm{n}=300$ volunteers).

\begin{tabular}{llcc}
\hline Test & Samples & Positives & Positivity (\%) \\
\hline Kato-Katz & 1 sample (1 slide) & 14 & 4.7 \\
Kato-Katz & 1 sample (2 slides) & 18 & 6.0 \\
Kato-Katz & 1 st \& 2nd samples (4 slides) & 21 & 8.0 \\
Kato-Katz & 1 st, 2nd, \& 3rd samples (6 slides) & 26 & 8.7 \\
POC-CCA assay & 1 sample & 82 & 27.3 \\
\hline
\end{tabular}

POC-CCA: point-of-care circulating cathodic antigen.

TABLE 3

Sensitivity, specificity, likelihood ratio, and predictive value of the POC-CCA urine assay.

\begin{tabular}{|c|c|c|c|c|c|}
\hline Gold standard & Sensitivity (CI) & Specificity (CI) & LR (CI) & PPV & NPV \\
\hline 1st \& 2nd samples / 4 slides & $52.4(32.4-71.6)$ & $75.3(69.5-80.3)$ & $2.1(1.33-3.3)$ & 13.7 & 6.3 \\
\hline Kato-Katz (AS) & $57.7(38.9-74.5)$ & $75.5(70.1-80.3)$ & $2.3(1.59-3.4)$ & 18.4 & 4.9 \\
\hline
\end{tabular}

POC-CCA: point-of-care circulating cathodic antigen; CI: confidence interval; LR: likelihood ratio; PPV: positive predictive value; NPV: negative predictive value; AS: any sample.

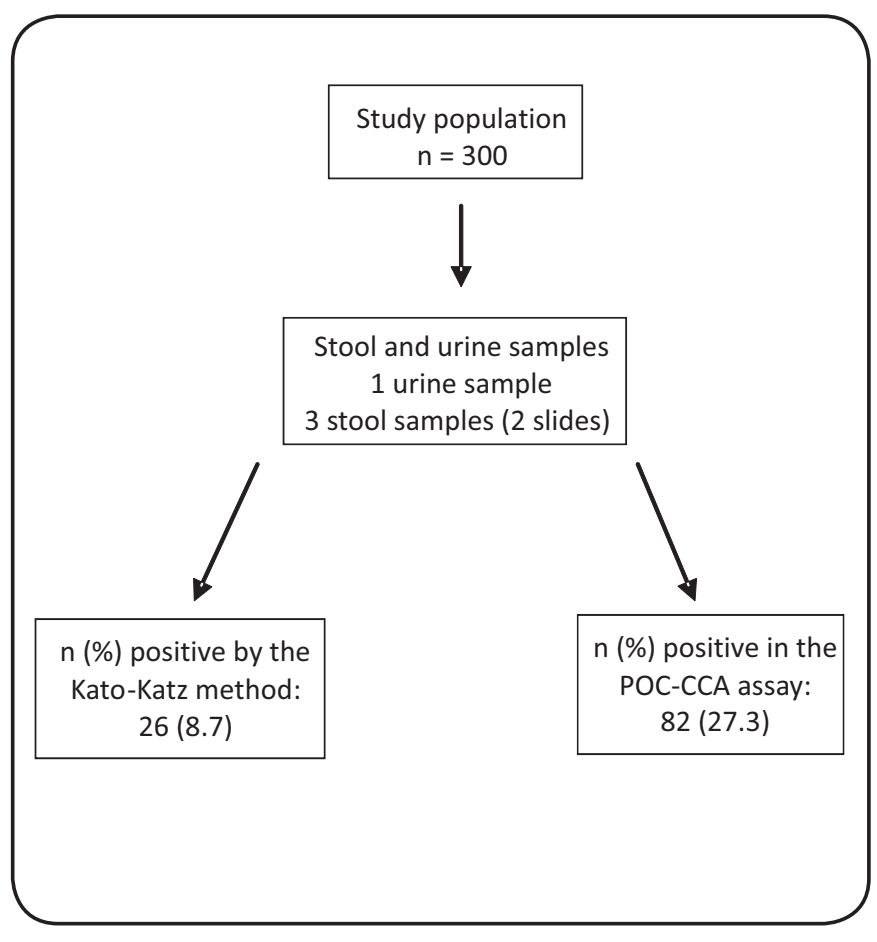

FIGURE 1 - Flowchart of the study. POC-CCA: point-of-care circulating cathodic antigen. pairs (stool positive and POC-CCA negative). A total of 55 participants were needed to reach a reliable result with a $5 \%$ error and $95 \%$ confidence interval. Five stool samples (two smears of each sample) on 5 consecutive days and three urine samples (one smear each) on 3 consecutive days were collected. Three $(5.9 \%)$ of 51 stool samples ( 480 slides) were positive for $S$. mansoni eggs. Five (45.5\%) out of the 11 Kato-Katz positives and POC-CCA negatives turned POC-CCA positive using three urine tests.

\section{Agreement between the tests}

The agreement between the results of the Kato-Katz smears and the POC-CCA assay was low for all comparisons in the 300 subjects examined $(\kappa=0.146)$.

\section{Morbidity of schistosomiasis}

Clinical and ultrasound examinations identified hepatosplenic schistosomiasis (characteristic periportal fibrosis, portal hypertension, and splenomegaly) in $2(1.1 \%)$ out of 181 patients. One patient, a 56-year-old man, had a positive stool examination for $S$. mansoni (46 eggs per gram of feces) and had a POC-CCA negative. The other patient, a 15-year-old boy, had three negative Kato-Katz examinations and one POC-CCA negative. The first patient received praziquantel $(50 \mathrm{mg} / \mathrm{kg}$ of 
body weight, oral single dose), and both are being followed up at the outpatient clinic at the University Hospital in Belo Horizonte, Minas Gerais, Brazil.

\section{DISCUSSION}

Based on the results, the POC-CCA test revealed more positive results for schistosomiasis than the Kato-Katz technique. Given the low prevalence of schistosomiasis in the area, morbidity was considered significant.

Most studies comparing the CCA assay to the KatoKatz technique were conducted in African countries, in individuals living in moderately to highly endemic areas for schistosomiasis $^{12,13,18-27}$. In a few reports conducted in areas of low prevalence in Africa, the comparisons were performed after repeated treatment with praziquante ${ }^{28}$. Reinfection may have occurred in these areas ${ }^{29}$. To our knowledge, this study is the first to evaluate the use of a rapid urine assay and schistosomiasis morbidity in an area of low prevalence in Brazil.

In schistosomiasis, immature worms during the acute phase or in recently reinfected cases may produce worm antigens (e.g., CCA) before eggs are excreted in the stool. This process may result in a positive result using the POC-CCA assay and a negative result using the Kato-Katz technique ${ }^{30-32}$.

Adriko et al..$^{23}$ evaluated the POC-CCA assay in an area of low $(8 \%)$ prevalence and found that the sensitivity values using two, four, and six Kato-Katz smears were $75 \%, 73 \%$, and $75 \%$, respectively, and the specificity values were $54 \%, 55 \%$, and $55 \%$, respectively. We found a lower sensitivity and higher specificity using one, two, four, and six smears; the sensitivity values were $64.3 \%, 61.1 \%, 58.3 \%$, and $55.5 \%$, respectively, and the specificity values were $74.5 \%, 74.8 \%, 75 \%$, and $75.6 \%$, respectively.

The sensitivity and specificity vary in areas of high prevalence. Studies have typically found high sensitivity and low to moderate specificity. For example, Shane et $\mathrm{a}^{21}$ reported that the sensitivity of the POC-CCA assay was $94.2 \%$ and the specificity was $59.4 \%$ in Kenya. In other studies using LCA, the sensitivity and specificity were increased ${ }^{13,21}$.

Colley et al. ${ }^{23}$ and Kittur et al. ${ }^{33}$ reported that the CCA assay could produce false negatives and false positives for $S$. mansoni. Indeed, our results showed that 11 (3.6\%) participants were positive using the Kato-Katz method but negative using the POC-CCA assay. It is interesting to note that 67 (22.3\%) participants had a positive POC-CCA urine assay and a negative Kato-Katz test. To further examine our findings, one of the study authors revisited the area and obtained 240 stool samples (for a total of 480 slides) from the 67 participants and found that 3 individuals (5.8\%) exhibited S. mansoni shedding of 12, 36, and 42 eggs per gram of feces. The argument is that, even in this context, the POC-CCA assay had a higher sensitivity for diagnosing $S$. mansoni infection than the KatoKatz method.

Some factors, such as diabetes mellitus, urinary tract infections, hematuria, alcohol abuse, use of diuretics, dehydration, and use of some prescription drugs (e.g., rifampin, which may change the color of the strip test), may alter the positivity of the rapid urine test, particularly in trace/weak positives. More studies are required to confirm these factors.

The POC-CCA assay showed poor agreement $(k=0.146)$ when the Kato-Katz technique was used as the gold standard. Similar findings have been described by other studies conducted in regions of very low prevalence and intensity of infection ${ }^{29}$. Day-to-day variability in host egg excretion and test limitations may explain these results.

In this study, the clinical and ultrasound examinations showed that only two patients had typical hepatosplenic schistosomiasis with the characteristic periportal fibrosis. The Brazilian Program for Schistosomiasis Control, which started in $1975^{33}$, was based on mass chemotherapy and its main objective was to reduce the prevalence of hepatosplenic schistosomiasis ${ }^{34,35}$. Most investigators, indeed, feel that the morbidity (hepatosplenic form) of schistosomiasis decreased. Of note is that even in low-endemic areas, such as the one reported above (prevalence of $8 \%$ ), severe cases were still found and they may be overlooked in similar areas. For example, one patient with hepatosplenic schistosomiasis, diagnosed during the abovementioned study, developed portal vein thrombosis, ascites, and large esophageal varices ${ }^{35}$.

Overall, the POC-CCA assay proved to be a useful test for diagnosing schistosomiasis in a low-endemic area. We are convinced that POC-CCA should be included in the Brazilian Program for Schistosomiasis Control and be liberated for clinical use. Nevertheless, improvements in diagnostic techniques, in areas of very low prevalence and intensity of infection, should be encouraged ${ }^{36}$.

More recently, after the WHO's appeal to define the value of the rapid urine test (POC-CCA) for schistosomiasis in lowendemic areas, Brazilian investigators have published papers on the subject ${ }^{37-39}$. Preliminary information is being evaluated, and other studies in Brazil are under way.

\section{Acknowledgments}

We offer our deepest thanks to the institutions that provided technical support for the development and implementation of this study; to Virginia Vilela Rabelo (Municipal Secretary of Pains) and Sandra Costa Drummond (Secretary of Minas Gerais State); to Alícia Aparecida Gonçalves Borges, Rísia Costa Nascimento, Beatriz da Costa Vieira, Francisco de Assis de Oliveira, and Silvana Romano; and to the Nature Publishing Group for the editorial assistance.

\section{Conflict of interest}

The authors declare that have no conflicts of interest.

\section{Financial support}

This work was supported by the Fundação de Amparo à Pesquisa do Estado de Minas Gerais, Conselho Nacional de Desenvolvimento Científico e Tecnológico, and Coordenação de Aperfeiçoamento de Pessoal de Nivel Superior. This work was supported in part by the Intramural Research Program of the National Institutes of Health (fellowship awarded). 


\section{REFERENCES}

1. Steinmann P, Keiser J, Bos R, Tanner M, Utzinger J. Schistosomiasis and water resource development: systematic review, meta-analysis, and estimates of people risk. Lancet Inf Dis. 2006;6(7):411-25.

2. Ministério da Saúde. Departamento de Vigilância Epidemiológica. Vigilância da Esquistossomose Mansoni: diretrizes técnicas/ Ministério da Saúde, Secretaria de Vigilância em Saúde, Departamento de Vigilância das Doenças Transmissíveis. 4. ed. Brasília: Ministério da Saúde; 2014. 141p.

3. Drummond SC, Silva LCS, Amaral RS, Sousa-Pereira SR, Antunes CM, Lambertucci JR. Morbidity of schistosomiasis mansoni in the state of Minas Gerais, Brazil. Mem Inst Oswaldo Cruz. 2006; 101(Supp 1):37-44.

4. Lambertucci JR. Schistosoma mansoni: pathological and clinical aspects. In: Jordan P, Webbe G, editors. Human schistosomiasis. 1st ed. Oxon, UK: Cab International Wallingford; 1993. p. 195-235.

5. Lambertucci JR, Cota GF, Pinto-Silva RA, Serufo JC, GerspacherLara R, Drummond SC, et al. Hepatosplenic schistosomiasis in field-based studies: a combined clinical and sonografic definition. Mem Inst Oswaldo Cruz. 2001;96(Suppl):147-50.

6. World Health Organization. Ultrasound in schistosomiasis: a practical guide to the standard use of ultrasonography for assessment of schistosomiasis-related morbidity. [Internet]. Geneva: World Health Organization; 2000. [cited 2017 May 22]. Available from: http://apps.who.int/iris/bitstream/10665/66535/1/TDR STR SCH 00.1.pdf

7. De Vlas SJ, Gryseels B. Underestimation of Schistosoma mansoni prevalences. Parasitol Today. 1992;8(8):274-7.

8. Utzinger J, Booth M, N'Goran EK, Muller I, Tanner M, Lengeler C. Relative contribution of day-to-day and intra specimen variation in faecal egg count of Schistosoma mansoni before and after treatment with praziquantel. Parasitology. 2001;122(Pt):537-44.

9. World Health Organization. The control of schistosomiasis: report of a WHO expert committee. Geneva: World Health Organization; 1984. [Internet]. [cited 2017 May 22]. Available from: http://apps. who.int/iris/bitstream/10665/39529/2/WHO_TRS_728_rus.pdf

10. Gomes LI, Enk MJ, Rabello A. Diagnosing schistosomiasis: where are we? Rev Soc Bras Med Trop 2014;47(1):3-11.

11. Enk MJ, Lima ACL, Drummond SC, Schall VT, Coelho PMZ. The effect of the number of stool samples on the observed prevalence and the infection intensity with Schistosoma mansoni among a population in an area of low transmission. Acta Trop. 2008;108 (2-3):222-8.

12. Legesse M, Erko B. Field-based evaluation of a reagent strip test for diagnosis of Schistosoma mansoni by detecting circulating cathodic antigen in urine before and after chemotherapy. Trans R Soc Trop Med Hyg. 2007;101(7):668-73.

13. Lamberton PHL, Kabatereine NB, Oguttu DW, Fenwick A, Webster JP. Sensitivity and specificity of multiple Kato-Katz thick smears and a circulating cathodic antigen test for Schistosoma mansoni diagnosis pre-and post- repeated-praziquantel treatment. PloS Negl Trop Dis. 2014;8(9):e3139.

14. World Health Organization. Elimination of schistosomiasis from low-transmission areas: report of a WHO informal consultation. Geneva: World Health Organization; 2009. [Internet] [cited 2017 May 22]. Available from: http://apps.who.int/iris/ bitstream/10665/70127/1/WHO_HTM_NTD_PCT_2009.2_eng.pdf

15. Pinto-Silva R, Queiroz LC, Azeredo LM, Silva LCS, Lambertucci JR. Ultrasound in schistosomiasis mansoni. Mem Inst Oswaldo Cruz. 2010;105(4):479-84.
16. Queiroz LC, Drummond SC, Matos ML, Paiva MB, Batista TS, Kansaon AZ, et al. Comparative randomized trial of high and conventional doses of praziquantel in the treatment of schistosomiasis mansoni. Mem Inst Oswaldo Cruz. 2010;105(4):445-8.

17. Dendukuri N, Joseph L. Bayesian approaches to modeling the conditional dependence between multiple diagnostic tests. Biometrics 2001;57(3):158-167.

18. Legesse M, Erko B. Field-based evaluation of a reagent strip test for diagnosis of Schistosoma mansoni by detecting circulating cathodic antigen in urine before and after chemotherapy. Trans R Soc Trop Med Hyg. 2007;101(7):668-73.

19. Ashton RA, Stewart BT, Petty N, Lado M, Finn T, Brooker S, et al. Accuracy of circulating cathodic antigen tests for rapid mapping of Schistosoma mansoni and Schistosoma haematobium infections in southern Sudan. Trop Med Inter Health. 2011;16(9):1099-103.

20. Coulibaly JT, Knopp S, N'Guessan NA, Silué KD, Furst T, Lohourignon LK, et al. Accuracy of urine circulating cathodic antigen (CCA) test for Schistosoma mansoni diagnosis in different settings of Cotê d' Ivoire. PLoS Negl Trop Dis. 2011;5(11):e1384.

21. Shane HL, Verani JR, Abudho B, Montgomery SP, Blackstocke AJ, Mwinzi PN, et al. Evaluation of urine CCA assays for detection of Schistosoma mansoni infection in western Kenya. Plos Negl Trop Dis. 2011; 5(1):e951.

22. Navaratnam AMD, Mutumba-Nakalembe MJ, Stothard JR, Kabatereine NB, Fenwick A, Sousa-Figueiredo JC. Notes on the use of urine CCA dipsticks for detection of intestinal schistosomiasis in preschool children. Trans R Soc Trop Med Hyg. 2012;106:619-22.

23. Colley DG, Binder S, Campbell C, King CH, TchuenTchunté LA, N'Goran EK, et al. Five country evaluation of point-of-care circulating antigen urine assay for the prevalence of Schistosoma mansoni. Am J Trop Med Hyg. 2013;88(3):426-32.

24. Coulibaly JT, N’Gbesso YK, Knopp S, N'Guessan NA, Silué KD, van Dam GF, et al. Accuracy of urine circulating antigen test for diagnosis of Schistosoma mansoni in preschool-aged children before and after treatment. Plos Negl Trop Dis. 2013;7(3):e2109.

25. Koukounari A, Donnelly CA, Moustaki I, Tukahebwa EM, Kabatereine NB, Wilson S, et al. A latent Markov modeling approach to the evaluation of circulating cathodic antigen strips for schistosomiasis diagnosis pre and post-praziquantel treatment in Uganda. Plos Comput Biol. 2013;9(12):e1003402.

26. Lodh N, Mwansa JCL, Mutengo MM, Shiff CJ. Diagnosis of Schistosoma mansoni without the stool: comparison of three diagnostic tests to detect Schistosoma mansoni infection from filtered urine in Zambia. Am J Trop Med Hyg. 2013;89(1):46-50.

27. Degarege A, Legesse M, Medhin G, Teklehaymanot T, Erko B. Dayto-day fluctuation of point-of-care circulating cathodic antigen test scores and fecal egg counts in children infected with Schistosoma mansoni in Ethiopia. BMC Infec Dis. 2014;14(3):1-7.

28. Sousa-Figueiredo JC, Betson M, Kabatereine NB, Stothard JR. The urine circulating cathodic antigen (CCA) dipstick: A valid substitute for microscopy for mapping and point-of care diagnosis of intestinal schistosomiasis. Plos Negl Trop Dis. 2013;7(1):e2008.

29. Adriko M, Standley J, Tinkitina B, Tukahebwa EM, Fenwick A, Fleming FM, et al. Evaluation of circulating cathodic antigen (CCA) urine cassette assay as a survey tool for Schistosoma mansoni in different transmission setting within Bugiri district, Uganda. Acta Trop. 2014;136(1):50-7.

30. Doenhoff MJ, Modha J, Lambertucci JR, McLaren DJ. The immune dependence of chemotherapy. Parasitol Today. 1991;7(1):16-18.

31. Lambertucci JR. Acute schistosomiasis mansoni: revisited and reconsidered. Mem Inst Oswaldo Cruz. 2010;105(4):422-35.1 
32. Lambertucci JR, Drummond SC, Voieta I, Queiróz LC, Pereira PPN, Chaves BA, et al. An outbreak of acute Schistosoma mansoni schistosomiasis in a non-endemic area of Brazil: A report on 50 cases, including five with severe clinical manifestations. Clin Infect Dis. 2013;57(1):e1-6.

33. Kittur N, Castleman JD, Campbell Jr CH, King CH, Colley DG. Comparison of Schistosoma mansoni prevalence of infection, as determined by the circulating cathodic antigen urine assay or by the Kato-Katz fecal assay: a systematic review. Am J Trop Med Hyg. 2016;94(3):605-10.

34. Machado PA. The Brazilian program for schistosomiasis control, 1975-1979. Am J Trop Med Hyg. 1982;31(1):76-86.

35. Lambertucci JR, Serufo JC, Gerspacher-Lara R, Rayes AA, Teixeira $\mathrm{R}$, Nobre V, et al. Schistosoma mansoni: assessment of morbidity before and after control. Acta Trop. 2000;77(1):101-9.
36. Lambertucci JR. Revisiting the concept of hepatosplenic schistosomiasis and its challenges using traditional and new tools. Rev Soc Bras Med Trop. 2014;47(2):130-6.

37. Caldeira K, Teixeira CF, Silveira MB, Fries LC, Romanzini J, Bittencourt HR, et al. Comparison of the Kato-Katz and Helmintex methods for the diagnosis of schistosomiasis in a low-intensity transmission focus in Bandeirantes, Paraná, southern Brazil. Mem Inst Oswaldo Cruz. 2012;107(5):690-2.

38. Siqueira LMV, Couto FFB, Taboada D, Oliveira AA, Carneiro NFF, Oliveira E, et al. Performance of POC-CCA in diagnosis of schistosomiasis mansoni in individuals with low parasite burden. Rev Soc Bras Med Trop. 2016;49(3):341-7.

39. Silveira AMS, Costa EGD, Ray D, Suzuki BM, Hsieh MH, Fraga $\mathrm{LAO}$, et al. Evaluation of the CCA immuno-chromatographic test to diagnose Schistosoma mansoni in Minas Gerais State, Brazil. PLoS Negl Trop Dis. 2016;10:e0004357. 